\title{
ON SOLVABILITY OF BOUNDARY VALUE PROBLEM FOR ELLIPTIC EQUATIONS WITH BITSADZE-SAMARSKII CONDITION
}

\author{
J.H. CHABROWSKI \\ The University of Queensland, \\ Department of Mathematics, \\ St. Lucia, 4067, Q1d, \\ Australia. \\ (Received January 21, 1987)
}

ABSTRAC'I'. In this paper we investigate the solvability of a non-local problen for a linear elliptic equation, which is also known as the boundary value liolliom with the Bitsadze-Samarskir condition. We prove the existence and uniqueness of a classical solution to this problem. In the final part of this paper we propose an $L^{2}$-approach which gives a rise to weak solutions in a weighted Sobolev space. The crucial point in proving the existence of weak solutions is a suitable modification of the Bitsadze--Samarskil condition.

KEY WORISS ANI PHRASES. Elliptic equations, non-local problems, Bitsazlzr-Samarski í condıtions.

1980 AMS SUR.JECT CLASSIFICATION CODES. 35J,35C,35R.

1. INTHODUCTION. In recent years several authors have studied the solvability of non-local problems for elliptic and parabolic equations [1-9]. The importance of non-local problems appears to have been first noted in the literature by Bitsadze-Samarskil. The problem studied in these papers constitutes a direct generalization of the classical boundary value problems. The most significant feature of nonlocal problems is that the boundary condition relates values of a solution on the boundary to its values on some part of the interior of the region. This type of the boundary value problem is often referred to as the boundary value problem with the Bitsadze-Samarskii condition [7],[8]. The problem (2.1), (2.2) discussed in this article arises from the mathematical description of some processes in a plasma (see paper [6] for full account of physical aspects of non-loral problems). 
The paper is organized as follows. In Section 2 we give the uniqueness and existence theorem of the classical solutions of the problem (2.1),(2.2). Our nethor is based on the maximum principle developed in papers [.1] and [5]. Sertion 3 contains a discussion of the solvability of the mon local problem for harmonic functions in a disc in $H_{2}$. The results of this section slightly improve the explicit formulae derived by Bitsadze iste [1] and [2]) for harmonic functions associated with some non-loca] problem. In a general case of a linear elliptir equation we reduce the problem :2.11,:2.2) to the solvability of the integral equation of the second kunl. The final sections 4 and 5 are devoted to the study of the non-lexil problem for a linear elliptic equation with a parameter, whose principal part is in a divergence form. This allows us to remove some restrictuons on the coefficient $\beta$ appearing in the boundary condtion (2.2). On the other hand this also suggests further extensions of the solvability of the problem $(2.1),(2.2)$ in a weighted Sobolev space $\tilde{w}^{l, 2}(Q)$. We adopt here the $I^{2}$-approach to the Dirichlet problem with $L^{2}$-boundary data from [13] and $[10]$.

2. UNIOUENLSS AND A PRIORI ESTIMATE.

We consider a linear equation of the elliptic type

$$
f u=\sum_{i, j=1}^{n} a_{i j}(x) b_{i j} u+\sum_{i=1}^{n} b_{i}(x) D_{i} u+c(x) u=f(x)
$$

in $Q$, where $Q$ is a bounded domain in $R_{n}$. The purpose of this paper is to investigate the following non-local problem: given continuous functions $h$ and $\beta$ defined on the boundary $\partial Q$ of $Q$ find a solution $u \in C^{2}(Q) \cap C(\bar{Q})$ satisfying the boundary condition

$$
u(x)-\beta(x) u(\phi(x))=h(x) \text { on } \partial Q \text {, }
$$

where $\phi$ is a given continuous mapping of $\partial Q$ into $Q$.

Throughout this section we make the following assumption

(i) The coefficients of the operator $L$ are bounded in $Q$ and there exists a constant $r>0$ such that

$$
\gamma|\xi|^{2} \leq \sum_{i, j=1}^{n} a_{i j}(x) \xi_{i} \xi_{j}
$$

for all $x \in Q$ and $\xi \in R_{n}$.

Moreover we assume that $Q$ satisfies an interior sphere condition at each point of to (see [11], p. 33-35).

The uniqueness of the problem $(2.1),(2.2)$ is a consequence of the strong maximum principle.

PROPOSITION 1. Let $|\beta(x)| \leq 1$ on $\partial Q$ and $c(x) \leq 0$ in $Q$ and suppose that 
either

or

$$
\text { (a) }-1 \leq \beta\left(x_{0}\right)<1 \text { at some point } x_{0} \in \partial Q
$$

(b) $c ! x_{1}$; $<0$ at some point $x_{1} \in Q$.

Then the problem $(2.1),(2.2)$ has at most one solution in $c^{2}(Q) \cap C(\vec{Q})$.

r'ROOF. It is sufficient to show that if $f(x) \equiv 0$ on $Q$ and $h(x) \equiv 0$ on $\partial Q$ then $u \equiv 0$ is the only solution of the problem (2.1),(2.2). It is clear that under rach of the assumptions (a) or (b) any constant solution must be identirally equal to 0 . If $u \notin 0$ then $u$ must be a non-constant solution and by the strong maximum principle ([11], Theorem 3.5 ) we may assume that

$$
u \because,=\max _{\bar{Q}} u(x) ; 0 \text { with } x_{2} \in \partial Q .
$$

If $\left.\beta^{\prime} \ddot{2}_{2}\right)=0$ we get a contradiction. Therefore it remains to consider two cases

$$
\text { (i) } 0<\beta\left(x_{2}\right) \leq 1 \text { and (ii) }-1 \leq \beta\left(x_{2}\right)<0 \text {. }
$$

In the first case $u\left(\phi\left(x_{2}\right)\right)=\frac{u\left(x_{2}\right)}{p\left(x_{2}\right)} \geq u\left(x_{2}\right)$, which is impossible since $\phi\left(x_{2}\right) \in Q$. In the second case (ii). we have

$$
u\left(\phi\left(x_{2}\right)\right)=\frac{u\left(x_{2}\right)}{-\bar{\beta}\left(x_{2}\right)}<0
$$

and by the strong maximum principle $u$ takes on a negative minimum at $x_{3} \in \partial Q$, that is

$$
u\left(x_{3}\right)=\min _{\bar{Q}} u(x)<0
$$

and we may assume that $\beta\left(x_{3}\right)<0$ since otherwise we get a contradiction. Hence

$$
u\left(\phi\left(x_{3}\right)\right)=\frac{u\left(x_{3}\right)}{\beta\left(x_{3}\right)}>0
$$

Now we distinguish two cases either

$$
u\left(x_{2}\right) \leq\left|u\left(x_{3}\right)\right| \text { or } u\left(x_{2}\right)>\left|u\left(x_{3}\right)\right|
$$

We show that both cases lead to a contradiction. Indeed, in the first case we have

$$
u\left(x_{2}\right) \leq\left|u\left(x_{3}\right)\right|=\left|\beta\left(x_{3}\right)\right|\left|u\left(\phi\left(x_{3}\right)\right)\right| \leq\left|u\left(\phi\left(x_{3}\right)\right)\right|=u\left(\phi\left(x_{3}\right)\right),
$$




$$
\left|u\left(x_{3}\right)\right| \cdot u\left(x_{2}\right)=\beta\left(x_{2}\right) u\left(\phi\left(x_{2}\right)\right) \leq\left|u\left(\phi\left(x_{2}\right)\right)\right| \text {. }
$$

Since both values $u\left(x_{3}\right)$ and $u\left(\phi\left(x_{2}\right)\right)$ are negative $u$ attains its negative minsmum at $\phi\left(x_{2}\right) \in Q$ and we arrive at a contradiction.

Inspertion of the prowf of Proposition 1 shows that the following version of the maximum principle holds true.

PROPOSITION 2. Suppose that $C(x) \leq 0$ in $Q$ and $0 \leq \beta(x) \leq 1$ on $\partial Q$. Let Lu $\leq 0 \quad(\geq 0)$ in $Q$, and $u(x)-\beta(x) u(\phi(x)) \geq 0(\leq 0)$ on $\partial Q$. Then $u(x) \geq 0(\leq 0)$ on $\vec{Q}$.

As an inmediale cunsequence we deduce an a priori estimate

THEOKEM 1. Suppose that $c(x) \leq-d$ in $Q$ and $0 \leq \beta(x) \leq \alpha$ where $d>0$ and $0<\alpha<l$ are constants. If $u$ is a solution of the problem (2.1),(2.2), then

PROOF. Let us define

$$
|u(x)|<\frac{1}{d} \sup _{Q}|f(x)|+\frac{1}{1-\alpha} \sup _{\partial Q}|h(x)| \text { for all } x \in \bar{Q} .
$$

$$
v(x)=u(x)-\frac{1}{d} \sup _{Q}|f(x)|-\frac{1}{1-\alpha} \sup _{\partial Q}|h(x)|,
$$

then we have

in $Q$ and

$$
L v=f-\frac{c}{d} \sup _{Q}|f(x)|-\frac{c}{1-\alpha} \sup _{\partial Q}|h(x)| \geq f+\sup _{Q}|f(x)| \geq 0
$$

$$
\begin{aligned}
v(x)-\beta(x) v(\phi(x))=h(x)-\frac{1}{d} \sup _{Q}|f(x)|-\frac{1}{1-\alpha} \sup _{\partial Q}|h(x)|+ \\
\quad+\frac{\beta(x)}{1-\alpha} \sup _{\partial Q}|h(x)|+\frac{\beta(x)}{\alpha} \sup _{Q}|f(x)| \leq \\
\leq\left(1-\frac{1}{1-\alpha}+\frac{\alpha}{1-\alpha}\right) \sup _{\partial Q}|h(x)|=0
\end{aligned}
$$

on $\partial Q$. Hemet by Proposition 2

$$
u(x) \leq \frac{1}{d} \sup _{Q}|f(x)|+\frac{1}{1-\alpha} \sup _{\partial Q}|h(x)|
$$

on $Q$. Similarly we can establish the inequality

$$
u(x) \geq-\frac{1}{d} \sup _{Q}|f(x)|-\frac{1}{1-\alpha} \sup _{\partial Q}|h(x)|
$$

on 0 , considering the auxiliary function 


$$
\text { wi: } u x:+\underset{0}{1} \sup |f(x)|+\underset{1-\alpha}{1} \sup |h(x)|
$$

REMARK 1 . If $c \equiv 0$ or $Q$ and $\beta(x)=1$ on $\partial Q$, then any two solutions of the problem (2.1),(2.2) differ by a constant.

3. EXISTENCL OH CIASSICAL SOLUTIONS.

We commence by considering a particular case of the non-local problem (2.1), (2.2) which consists of finding a harmonic function $u$ on $B(0,1)$ and satisfyine the houndary condition

$$
u^{\prime} z^{\prime}-\beta u(\phi(z))=h(z) \text { on } \partial B(0,1) \text {, }
$$

where $B(0,1)$ is an open disc in $R_{2}$ of radius $l$ centred at $0, \beta$ is a constant in the interval $[-1,1]$ and $h$ is a continuous function on $\partial B(0,1)$. The mapreng $\phi$ is given by $\phi(z)=\phi_{*}(\delta z)$ with $0<\delta<1$, where $\phi_{*}$ is a univalent analytic function on $B(0,1)$ such that $\left|\phi_{*}(z)\right| \leq 1$ in $B(0,1)$ and $\phi_{*}(0)=0$. By virtue of Schwarz's lemma we have

$$
\left|\phi_{*}(z)\right|<|z| \text { for all } z \in B(0,1) \text {. }
$$

The function $\phi$ maps disc $B(0,1)$ conformally and univalently onto certain set contained in $B(0,1)$. Letting $\phi_{0}(z)=z$ and $\phi_{k}(z)=\phi\left(\phi_{k-1}(z)\right)$ for $k=1,2, \ldots$ we have

$$
\left|\phi_{k}(z)\right|<\sigma^{k}|z| \text { in } \mathrm{B}(0,1) \text {, }
$$

for $k=1,2, \ldots$. Since $u$ and $u(\phi(z))$ are harmonic functions we have the following representation formula

$$
u(z) \cdots \beta u(\phi(z))=\operatorname{Re} \frac{1}{\pi i} \underset{\partial B(0,1)}{\int}\left[\frac{1}{t-z}-\frac{1}{2} \bar{t}\right] h(t) d t \equiv F(z)
$$

Suppose first that $-1<\beta<1$. Iterating (3.4) we get

$$
u(z)=\beta^{n} u\left(\phi_{n}(z)\right)+\sum_{k=1}^{n} \beta^{k-1} F\left(\phi_{k-1}(z)\right) .
$$

It follows from (3.3) that

$$
\begin{aligned}
\left|F\left(\phi_{n}(z)\right)\right| & =\left|\operatorname{Re} \frac{1}{2 \pi} \bar{i} \int_{\partial B(0,1)}^{s} \frac{t+\phi_{n}(z)}{t-\phi_{n}(z) \bar{t}} h(t) d t\right| \leq \\
& <\frac{1+\delta}{2 \pi(1-\bar{l})} \int_{0}^{\pi}\left|h\left(e^{i s}\right)\right| d s
\end{aligned}
$$

for $n=1,2, \ldots$ and consequently letting $n \rightarrow \infty$ in (3.5) we obtain 


$$
\left.u_{i} z\right)=\sum_{n=1}^{\infty} \beta^{n-1} F\left(\phi_{n-1}(z)\right)
$$

uniformly on $\overline{\mathrm{P}}(\mathrm{O}, \overline{1})$.

L. 1 us now consider the case $\beta=-1$. It follows from (3.4) that

$$
2 u(0)=\operatorname{Re} \frac{1}{\pi i} \quad \int_{\partial B(0,1)} \frac{h(t)}{2 t} d t
$$

and the functional equation (3.4) can be written in the form

$$
\begin{aligned}
u(z)+u(\phi(z))-2 u(0)=\operatorname{Re} \frac{1}{\pi} \bar{i} \int & \int_{\partial B(0,1)}\left[\bar{t}-\bar{z}-\frac{1}{2} \bar{t}-\frac{1}{2} \bar{t}\right] h(t) d t \\
= & \operatorname{Re} \frac{1}{\pi} \bar{i} \underset{\partial B(0,1)}{\int} \frac{h(t)}{t\left(\frac{t}{t}-z\right)} d t \cdot z \equiv \operatorname{Re}[\Phi(z) \cdot z] .
\end{aligned}
$$

Iterating the last equation we obtain

and

$$
u(z)=u\left(\phi_{2 n}(z)\right)+\sum_{j=0}^{2 n-1}(-1)^{j} \operatorname{Re}\left[\Phi\left(\phi_{j}(z)\right) \phi_{j}(z)\right]
$$

$$
u(z)=2 u(0)-u\left(\phi_{2 n-1}(z)\right)+\sum_{j=0}^{2 n-2}(-1)^{j} \operatorname{Re}\left[\Phi\left(\phi_{j}(z)\right) \phi_{j}(z)\right] .(3.8)
$$

It is easy to see that

$$
\left|\Phi\left(\phi_{n}(z)\right)\right| \leq-\frac{\delta}{\pi\left(\frac{1}{-\sigma}\right)} \int_{0}^{2 \pi}\left|\mathrm{h}\left(\mathrm{e}^{\mathrm{is}}\right)\right| \mathrm{ds}
$$

for all $n=1,2, \ldots$ and $z \in \bar{B}(\overline{0}, \overline{1})$. Since $\left|\phi_{j}(z)\right|<\delta^{j}$ in $\bar{B}(\overline{0}, \overline{1})$ the series $\sum_{j=0}^{\infty}(\cdot 1)^{j} \operatorname{Re}\left[\phi\left(\phi_{j}(z)\right) \phi_{j}(z)\right]$ converges uniformly on $\bar{B}(\overline{0}, \overline{1})$. Letting $n \rightarrow \infty$ in (3.7) and (3.8) we obtain the same limit in both cases

$$
u(z): u(0)+\sum_{j-0}^{\infty}(-1)^{j} \operatorname{Re}\left[\Phi\left(\phi_{j}(z)\right) \phi_{j}(z)\right]
$$

and invoking (3.6) we get that

$$
u(z)=\operatorname{Re} \frac{1}{4 \pi} \int_{\partial B(0,1)} \frac{h(t)}{t} d t+\sum_{j=0}^{\infty}(-1)^{j} \operatorname{Re}\left[\Phi\left(\phi_{j}(z)\right) \phi_{j}(z)\right] .
$$

Finally let $\beta=1$, then

$$
u(z)-u(\phi(z))=\operatorname{Re} \frac{1}{\pi} \bar{i} \underset{\partial B(0,1)}{\int}\left[\bar{t}-\bar{z}-\frac{1}{2} \bar{t}\right] h(t) d t
$$

By Remark 1 any two solutions differ by a constant. If $z=0$, then 


$$
0-u(0)-u(\phi(0))=\operatorname{ke} \frac{1}{\pi i} \int_{\partial B(0,1)} \frac{f(t)}{t} d t,
$$

which gives; a necessary and sufficient condition for the solvability of the problem $(3.1)$. Hence

$$
u(z)-u(\phi(z))=\operatorname{Re}[\Phi(z) z]
$$

Iterating this functional equation we obtain

$$
u(z)=u\left(\phi_{n}(z)\right)+\sum_{j=1}^{n \cdot-1} \operatorname{Re}\left[\Phi\left(\phi_{j}(z)\right) \phi_{j}(z)\right]
$$

Letting $n \rightarrow \infty$ we obtain

$$
u(z)=u(0)+\sum_{j=1}^{\infty} \operatorname{Re}\left[\Phi\left(\phi_{j}(z)\right) \phi_{j}(z)\right] .
$$

To determine a solution in a unique way we may impose an additional condition $u(0)=C$, where $C>0$ is a given constant. The case $\beta=1$ was considered by Bitsadze in [1] and [2] under the assumption that $h$ is Holder continuous on aQ.

In a general case we reduce the problem (2.1),(2.2) to the Fredholm integral equation of the second kind.

THEOREM 2. Suppose that the assumptions of Proposition 1 hold and that $v_{i j}^{2} a_{i j}(i, j=1, \ldots, n), D_{i} b_{i}(i=1, \ldots, n)$ and $c$ are Holder continuous on $\bar{Q}$. Then the problem $(2.1),(2.2)$ admits a unique solution $u$ in $C^{2}(Q) \cap c(\bar{Q})$.

PROOF. We try to find a solution in the form

$$
u(x)=\int_{\partial Q} \frac{d G(x, y)}{d n_{y}} v(y) d S y-\int_{Q} G(x, y) f(y) d y,
$$

where $v \in(:(\partial \theta)$ is to be determined, $G$ is the Green function for the operator $L$ and $\frac{d G}{d n_{y}}$ denotes the conormal derivative. The boundary condition (2.2) leurts to the fredholm integral equation of the second kind.

$$
\begin{gathered}
v(x)-\int_{d Q} \beta(x) \quad \underset{d n_{y}}{\operatorname{dg}\left(x_{2} y\right)} v(y) d S_{y}=h(x)+ \\
\quad+\int_{Q} \beta(x) G(\phi(x), y) f(y) d y .
\end{gathered}
$$

Since $\phi(\partial Q)=Q$ the kernel $\beta(x) \frac{\mathrm{dG}(\phi(x), y)}{\mathrm{dn} y}$ is continuous function on $\partial Q \times \partial Q$. Ly Proposition 1 the homogeneous equation corresponding to (3.10) has only trivial solution. Hence by the Fredholm alternative there exists 
a unique solution $v \in L^{2}(\partial \theta)$ which by the continuity of the kernel belongs to CraQ'. Consequently the formula (3.9) gives a solution to the problem (2.1), (2.2).

4. ENERGY ESTINATE.

In this sertion we consider the elliptic equation in the form

$$
\begin{aligned}
M u+\lambda u & =-\sum_{i, j=1}^{n} D_{i}\left(a_{i j}(x) D_{j} u\right)+\sum_{i=1}^{n} b_{i}(x) D_{i} u+c(x) u+ \\
& +\lambda u=f(x) \text { in } Q,
\end{aligned}
$$

with the boundary condition (2.2).

Throughout this section we assume that $\beta$ is a continuous function on $\partial Q$ and $\phi: \partial Q \rightarrow Q$ is a $C^{1}$-mapping with the positive Jacobian. Further we assume that $a_{i j}, b_{i} a_{i j}, b_{i}(i, j=1, \ldots, n) c$ and $f$ are Holder continuous on $Q$ and that $Q$ is a bounded domain with the boundary of class $C^{2}$.

The (b)jestive of this section is to show that the problem (4.1), (2.2) has a unique solution for large values of the parameter $\lambda$.

$$
\begin{aligned}
& \text { For small } \delta>0 \text { we define } Q_{\sigma}=Q \cap\{x ; \underset{y \in \partial Q}{\min }|x-y|>\delta\} \\
& \text { According to Lemma } 14.16 \text { in }[11] \quad \text { (p. 355), the distance }
\end{aligned}
$$

$r(x)=\operatorname{dist}(x, \partial Q)$ belongs to $c^{2}\left(\bar{Q}-Q_{\sigma_{0}}\right)$ if $\delta_{0}$ is sufficiently small. Denote by $\rho(x)$ the extension of the function $r(x)$ into $\bar{Q}$ satisfying the following properties

$$
\rho ! x)=r(x) \text { for } x \in \ddot{Q}-Q_{\sigma_{0}}, \rho \in C^{2}(\bar{\theta}), \rho(x),{ }^{3 \delta_{0}}-{ }^{-} \text {in } Q_{\sigma_{0}},
$$

${ }^{-1} r(x) \leq p(x) \leq \gamma_{1} r(x)$ in $Q$ for some constant $\gamma_{1}>0, \partial Q_{\delta}=\{x ; \rho(x)=$ $\delta$ ) for $\delta \in\left(0, \delta_{n}\right)$ and finally $\partial Q=\{x ; \rho(x)=0\}$.

THEOREM 3. There exist positive constants $\lambda_{0}, C$ and $d$ such that if $u$ is a solution in $\left(:^{2}(Q) \cap C(\bar{Q})\right.$ of the problem $(4.1),(2.2)$ for $\lambda \geq \lambda_{0}$ then

$$
\begin{gathered}
\int_{Q}^{\int|v u(x)|^{2} r(x) d x+\int_{Q} u(x)^{2} r(x) d x+\sup _{0<\delta \leq d} \int Q_{\delta} u(x)^{2} d S_{x} \leq} \\
\leq C\left(\int_{\partial Q} h(x)^{2} d S_{x}+\int_{Q} f(x)^{2} d x\right) .
\end{gathered}
$$

Proof. We follow the proof of Theorem 5 in [13]. Nultiplying (4.1) by 


$$
v(x)= \begin{cases}u(x)(p(x)-\sigma) & \text { on } Q_{\delta} \\ 0 & \text { on } Q-Q_{\delta}\end{cases}
$$

and integrating by parts we obtain

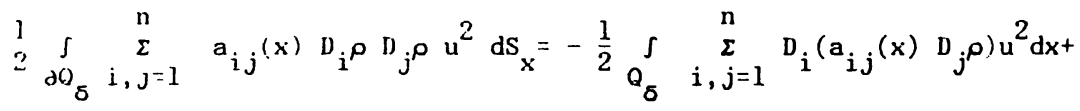

$$
\begin{aligned}
& 1 \int_{Q} \sum_{i=l}^{n} b_{1}(\lambda) D_{j} u \cdot u(\rho \delta) d x+\int_{Q_{\delta}}(c(x)+\lambda) u^{2}(\rho-\delta) d x-\int_{Q_{\delta}} f u(\rho \delta) d x .
\end{aligned}
$$

Applying Holder's inequality we easily obtain

$$
\begin{aligned}
\sup _{0 . \delta \leq d} & \int_{j Q_{\delta}} u^{2} d S_{x} \leq C_{1}\left[\int_{Q}|D u|^{2} \rho d x+\int_{Q} u^{2} d x+\right. \\
& \left.+\lambda \int_{Q} u^{2} \rho d x+\int_{Q} f^{2} d x\right]
\end{aligned}
$$

where $d$ and $c_{1}$ are positive constants.

Similarly

$$
\int_{Q}|u u|^{2} \rho d x+\lambda \int_{Q} u^{2} \rho d x \leq C_{2}\left[\int_{Q} u^{2} d x+\int f_{Q}^{2} d x+\int u_{\partial Q}^{2} d S x\right]
$$

for some $c_{2}, 0$. It follows from (2.2) that

$$
\begin{aligned}
& \int_{0}|n u|^{2} \rho d x+\lambda \int_{Q} u^{2} \rho d x \leq C_{3}\left[\int_{Q} u^{2} d x+\int_{Q} f^{2} d x+\right. \\
& \left.+\int_{0 Q} h^{2} d S_{x}+\int_{\partial Q} u(\phi(x))^{2} d S_{x}\right]
\end{aligned}
$$

where $r_{: 3} 0$. The estimates (4.2) and (4.4) yield that

$$
\begin{aligned}
& \int_{Q}|\mathrm{Du}|^{2} \rho \mathrm{dx}+\lambda \int_{Q} \mathrm{u}^{2} \rho \mathrm{dx}+\sup _{0<\delta<\mathrm{d}} \int_{\partial Q} \mathrm{u}^{2} \mathrm{dS} \leq \\
& \leq \mathrm{C}_{4}\left[\int_{Q} \mathrm{u}^{2} \mathrm{dS} \mathrm{x}_{\mathrm{x}}+\int_{Q} \mathrm{f}^{2} \mathrm{dx}+\int_{\partial Q} \mathrm{~h}^{2} \mathrm{dS}_{\mathrm{x}}+\int_{\partial Q} \mathrm{u}(\phi(\mathrm{x}))^{2} \mathrm{dS} \mathrm{S}_{\mathrm{x}}\right]
\end{aligned}
$$

for some $C_{4}>0$. Since $\phi$ is a $\mathrm{C}^{1}$-mapping with the positive Jacobian it is obvious that

$$
\left.\int_{\partial Q} u(p(x))^{2} d S_{x} \leq C_{5} \underset{\phi(\partial Q)}{\int} u^{2} d S_{x} \leq C_{6} \int_{Q_{\phi}}|D u|^{2} d x+\int_{Q_{\phi}} u^{2} d x\right],
$$

where $\mathrm{C}_{5}>0$ and $\mathrm{C}_{6}>0$ are constants and $Q_{\phi}$ is a domain containing $\phi(\partial Q)$ with dist $\left(Q_{\phi}, \partial Q\right)>0$. Consequently by virtue of the Caccioppoli inequality 
we have

$$
\int_{j Q} u(\phi(x))^{2} d S_{x} \leq C_{7}\left[\int_{Q} u^{2} d x+\int_{Q} f^{2} d x\right]
$$

for some $\mathrm{C}_{7}>0$. We now observe that

$$
\int_{0} \mathrm{u}^{2} \mathrm{dx} \leq \frac{1}{\mathrm{~d}} \int_{Q} \int_{Q} \mathrm{u}^{2} \rho \mathrm{dx}+\mathrm{d} \sup _{0<\delta \leq d} \int_{\partial Q} \mathrm{u}^{2} \mathrm{~d} \mathrm{~s}_{\mathrm{x}},
$$

where $d_{1}=\inf _{0} \rho(x)$. Choosing $d$ sufficiently small and $\lambda$ sufficiently $\mathrm{O}_{\mathrm{d}}$

large we easily derive the desired estimate from $(4.5),(4.6),(4.7)$ and (4.8).

Repeating the argument of Theorem 2 we deduce the following

THEOIREM 4. There exists a positive constant $\lambda_{0}$ such that for every $\lambda \geq \lambda_{0}$ the problem (4.1),(2.2) admits a unique solution in $C^{2}(Q) \cap C(\bar{Q})$.

5. WEAK SOLUTIONS.

The energy estimate from Section 4 shows that one can expect solutions of the problem $(4.1),(2.2)$ in a weighted Sobolev space defined by

$$
\ddot{W}^{1,2}(Q)=\left\{u \in W_{1 \text { oc }}^{1,2}(Q) ; \int_{Q}|\mathrm{Du}(x)|^{2} r(x) d x+\int_{Q} u(x)^{2} d x<\infty\right\}
$$

and equiperd with the norm

$$
\left.\left.|| u\right|_{w} ^{2}\right], 2=\int_{0}\left[|D u(x)|^{2} r(x)+u(x)^{2}\right] d x .
$$

We reall briefly that a function $u$ is said to be a weak (generalized) solution of (4.1) if $u \in W_{10 c}^{1,2}(Q)$ and it satisifies

$$
\int_{0}\left[\sum_{i, j=1}^{n} a_{i j} D_{i} u v v_{j} v+\sum_{i=1}^{n} b_{i} D_{i} u \cdot v+(c+\lambda) u v\right] d x=\int_{a} f v d x(5.1)
$$

for each $v \in W^{1,2}(Q)$ with compact support in $Q$.

To proceed further we need some terminology. It follows from the regularity of the boundary $\partial Q$ that there exists a number $\sigma_{0}$ such that for $\delta \in\left(0, \delta_{0}\right)$ the domain $Q_{\delta}$ (defined in Section 4) with the boundary ${ }^{\partial} Q_{\delta}$ possesses the following property: to each $x_{0} \in \partial Q$ there exists a unique point $x_{\delta}\left(x_{n}\right) \in \partial Q_{\delta}$ such that $x_{\delta}\left(x_{0}\right)=x_{0}-\delta \nu\left(x_{0}\right)$, where $\nu\left(x_{0}\right)$ is the outward normal to $\partial Q$ at $x_{0}$. The above relation gives a one-to-one mapping of class $c^{1}$, of $\partial Q$ onto $\partial Q_{\delta}$. 
It is known that elements of the space $\tilde{w}^{1,2}(Q)$, in general, do not have triares on the boundary $\partial Q$ (see [12]). However, by Theorem 4 in [13], if $\left.u \in W^{(, 2}, 0\right)$ is a solution of (4.1) then there exists a function $\zeta \in L^{2}(u 0)$ such that

$$
\lim _{\delta \rightarrow 0} \int_{\partial Q}\left[u\left(x_{\delta}\right)-s(x)\right]^{2} d S_{x}=0 .
$$

Thrrime, as in the paper [13], we adopt the following $L^{2}$-approach to the $1, r(1)+m(4.1),(2.2)$.

Let $h \in L^{2}(d Q)$. A weak solution $u \in W_{l o c}^{1,2}(Q)$ of $(4.1)$ is a solution of the non-local problem with the boundary condition (2.2) if

$$
\lim _{\delta \rightarrow 0} \int_{\partial Q}\left[u\left(x_{\delta}\right)-\beta(x) u(\phi(x))-h(x)\right]^{2} d s_{x}=0
$$

It follows from Theorem $l$ in [13] that if $u \in w_{10 c}^{1,2}(Q)$ is a solution of the problem (4.1),(2.2) (with the boundary condition (2.2) understood in the sense of $(5.2)$ then $u \in \tilde{w}^{1,2}(Q)$. We mention also that $u(\phi(x))$ is understood in the sense of trace, which is well defined since $u \in w_{l o c}^{1,2}(Q)$ (see $[1.1]$, ihap. C).

We now are in a position to establish the existence result in $\tilde{w}^{l, 2}(Q)$ of the problosn (1.1),:2.2).

THEOREM 5. Let $h \in L^{2}(\sigma 0)$. Then there exists a positive constant $\lambda_{0}$ such that for $\lambda \geq \lambda_{0}$ the problem $(4.1),(2.2)$ in $W_{10 c}^{1,2}(Q)$ admits a unique solution.

PROOF. Let $h_{\mathrm{in}}$ be a sequence in $\mathrm{C}^{\mathrm{l}}(\partial Q)$ such that $\lim _{\mathrm{m} \rightarrow \infty} \int_{\partial Q}\left(\mathrm{~h}_{\mathrm{m}}-\mathrm{h}\right)^{2} \mathrm{dS} \mathrm{x}_{\mathrm{X}}=0$. Let $\lambda_{0}$ he a constant from Theorem 4 and assume that $\lambda \geq \lambda_{0}$. For each $m \geq 1$ Theorem 4 guarantees the existence of the unique solution $u_{m} \in C^{2}(Q) \cap C(\bar{\theta})$ of the problem (4.1),(2.2) with $h=h_{m}$. Moreover we have for earh $u_{m}$

$$
\int_{Q}\left|D u_{m}\right|^{2} r d x+\int_{Q} u_{m}^{2} d x \leq C\left(\int_{Q} f^{2} d x+\iint_{\partial Q} h_{m}^{2} d S\right),
$$

where $C ; 0$ is a constant independent of $m$. Since the sequence $u_{m}$ is bounded in $\tilde{w}^{1,2}(0)$, there exists a subsequence, which we relabel as $u_{m}$, converging weakly in $\tilde{w}^{1,2}(Q)$ to a function $u$. By Theorem 4.11 in [15], $\tilde{w}^{1,2}(Q)$ is compactly embedded in $L^{2}(Q)$ and therefore we may assume that $u_{m}$ 
converges: $f(1)$ u in $l^{2}(Q)$. It is sbvious that $u$ is a reak solution of (4.1). By 'lheorell 1 in [13] $u$ has a trace $s \in L^{2}(\partial Q)$ in the sense of $L^{2}$-conwergence, that is

$$
\lim _{\delta \rightarrow 0} \int_{u Q}\left[u\left(x_{\delta}\right)-s(x)\right]^{2} d S_{x}=0
$$

To complete the proof we show that $s(x)=\beta(x) u(\phi(x))+h(x)$ a.e. on $\partial Q$. Let $\Phi \in c^{1}(0)$. It is easy to show $\Phi(x) \rho(x)$ is a legitimate test function in (5.1) anl integrating hy parts we obtain

$$
\begin{aligned}
& \int \zeta \Phi_{i, j=1}^{\sum} a_{i j} D_{i} \rho n_{j} \rho d S_{x}=-\int_{Q} \sum_{i, j=1}^{n} D_{i}\left(a_{i j} v_{j} \rho \Phi\right) u d x+ \\
& \cdot \int_{Q} \sum_{i=1}^{n} b_{i} v_{1} u \Phi\left(x+\int_{Q}(c+\lambda) u \Phi \rho d x-\int_{Q} r \Phi u \rho d x \equiv\right. \\
& \equiv f(u) .
\end{aligned}
$$

Similarly

$$
\int_{j()}\left[h_{m}(x)+\beta(x) u_{m}(\phi(x))\right] \sum_{i, j=1}^{n} a_{i j} D_{i} \rho D_{j} \rho d S_{x} \equiv F\left(u_{m}\right) .
$$

Aprlying the estinate (4.6) and the obvious analogue of the energy estimate $t_{0} u_{p}-u_{q}$ we obtain

$$
\begin{aligned}
& \int_{Q Q}\left[u_{p}(\phi(x))-u_{q}(\phi(x))\right]^{2} d S_{x} \leq C\left[\int_{Q_{\phi}}\left|D u_{p}-D u_{q}\right|^{2} d x+\right. \\
& \left.+\int_{Q}\left|u_{p}-u_{q}\right|^{2} d x\right] \leq \frac{C}{k} \int_{Q}\left|D u_{p}-D u_{q}\right|^{2} \rho d x+C \int_{Q}\left|u_{p}-u_{q}\right|^{2} d x \leq \\
& \leq C ; \int_{\partial Q}\left|h_{p}-h_{q}\right|^{2} d S_{x},
\end{aligned}
$$

where $\left.k \cdot \inf _{\tilde{Q}} \rho_{i}\right)$ and $\tilde{C}>0$ is a constant independent of $p$ and $q$. Hence $\bar{Q}_{\phi}$

by the continuity of weak solutions on $Q$ we may assume that $u_{m}(\phi(x)) \rightarrow u(\phi(x))$ as $m \rightarrow \infty$ in $L^{2}(\partial Q)$. Combining this with the fact that $F\left(u_{m}\right) \rightarrow F(u)$ as $m \rightarrow \infty$ we deduce from (5.3) and (5.4) that $\zeta(x)=\beta(x) u(\phi(x))+h(x)$ a.e. on $\partial Q$ and this completes the proof.

REMARK 2. It is worth noting that the non-local problem of the type (4.1),(2.2) has been studied in [9] for the higher order elliptic equations. The corresponding boundary datum $h$ in [9] belongs to the space $H^{\frac{1}{2}}(\partial Q)$. Since $H^{\frac{1}{2}}(\partial Q)$ is a proper subspace $L^{2}(\partial Q)$, Theorem 5 cannot be deduced from the results of the paper [9]. 
REFERENCES.

1. BITSAIIZE, A.V., 'On the theory of nonlocal boundary value problems', Sov1:1 Milt. Hok1. 30(1) (1981), 8-10.

2. BITSADZF, A.V., 'On a class of conditionally solvable non-local boundary value problems for harmonic functions', Soviet Mat. Dokl. 31(1) (1985), 91-94.

3. BITSADZE, A.V. and SAMARSKIİ, A.A., 'On some simple generalizations of linear elliptic boundary problems', Soviet Math. Dokl. 10(2) (1969), $398-400$.

4. CHABLOWSKL, J., 'On non-local problems for parabolic equations', Nagnya Math. J., 93 (1984), 109-131.

5. CHABROWSKI, J., 'On the non-local problem with a functional for parabolic equation', Funkcialaj Ekvacioj, 27(1) (1984), 101-123.

6. SAMAKSKII, A.A., 'Some problems in differential equation theory', Differenrial'nye Uravneniya, 16(11), (1980), 1925-1935.

7. SKUPACZEVSKI, A.L., 'Solvability of elliptic problems with BitsadzeSamiasili boundary conditions'. Differencial'nye Uravmeniya, 21 (4) (1985), 701-706.

8. SKLin(aLVSKI, A.l., 'Non-loral elliptic problems with a piarameter', Mitt. USSH Sb. 49(1) (1984), 197-206.

9. SkHBAC\%VVSKI, A.L., 'Sume non-local elliptic boundary-value problems', Differencial'nye Uravneniya 18(9) (1982), 1590-1599.

10. MIKHAILOV, V.P., 'Boundary values of elliptic equations in domains with a smooth boundary', Mat Sb. 101 (143) (1976), 163-188.

11. (illBAKK, $P$ ' and THUDINGEK, N.S., 'Elliptic partial differential equations of second order', Grundlehrnen der Mathematischen Wirssensichaften 224, Springer-Verlag, Berlin-Heidelberg-New York-Tokyo, 1983 (ivecond Edition).

12. POULSLI, libbe Thue, 'Boundary value properties connected with some improper llirichlet integrals', Math. Scand. 8 (1960), 5-14.

13. CHABHOWSKI, J. and THOMPSON, B., 'On the boundary values of the solutions of linear elliptic equations', Bull. Austral. Math. Soc. 27 (1983), 1-30.

14. KUFNEK, A., JOHN, O. and FUĆIK, S. 'Function spaces', Leyden, Noordhoff, Prague, Academia, 1977.

15. MEYER, R.D., 'Some embedding theorems for generalized Sobolev spaces and applications to degenerate elliptic differential operators', J. Math. Mech. 16 (1967), 739-760. 


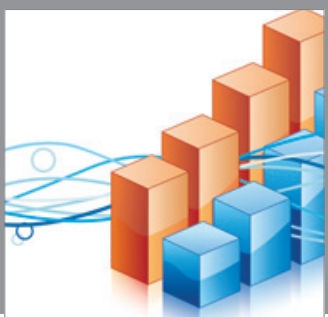

Advances in

Operations Research

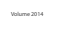

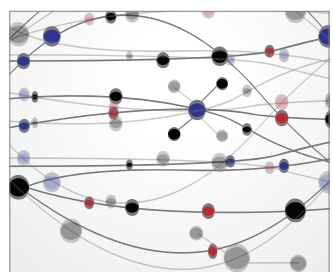

\section{The Scientific} World Journal
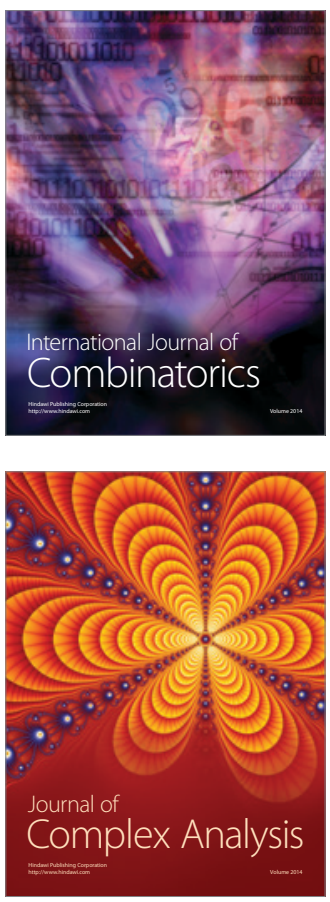

International Journal of

Mathematics and

Mathematical

Sciences
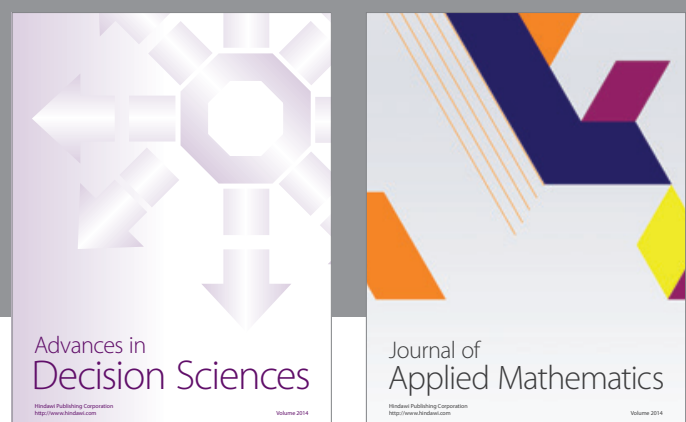

Journal of

Applied Mathematics
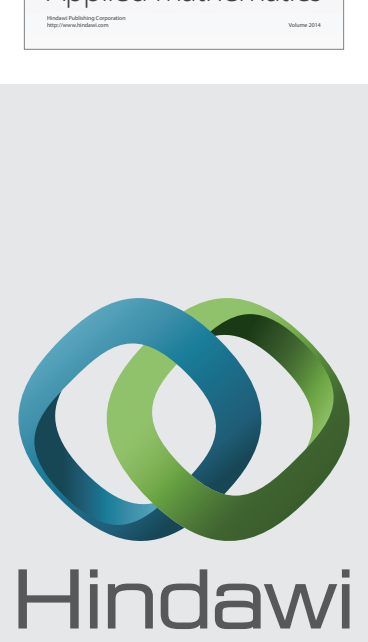

Submit your manuscripts at http://www.hindawi.com
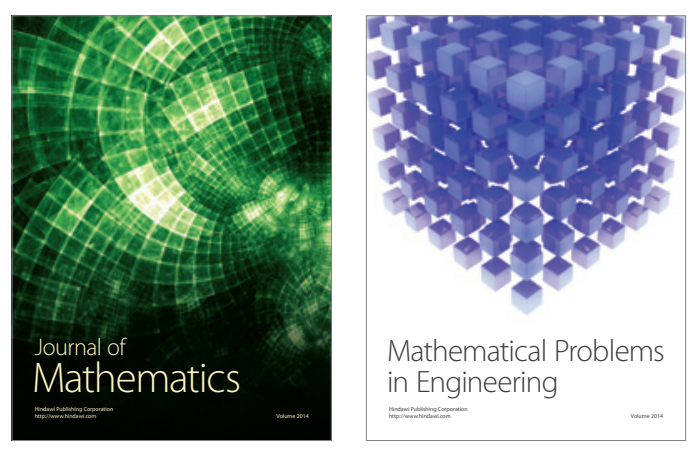

Mathematical Problems in Engineering
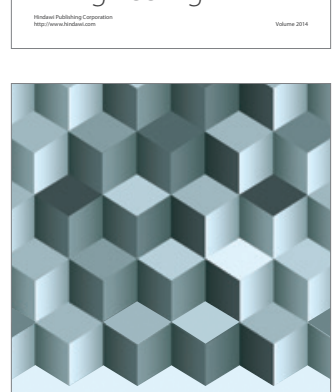

Journal of

Function Spaces


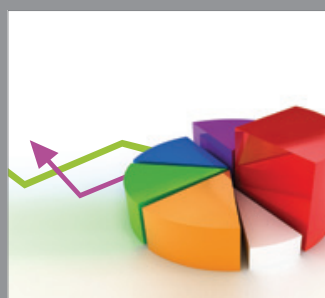

ournal of

Probability and Statistics

Promensencen
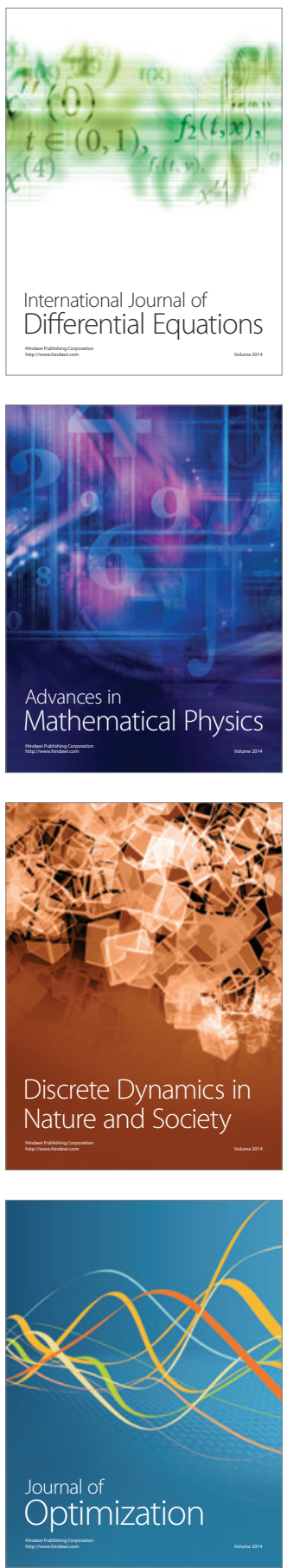